\title{
GENERALISED HARMONIC ANALYSIS OF A CLASS OF NONSTATIONARY RANDOM FIELDS
}

\author{
V. V. ANH and K. E. LUNNEY \\ (Received 15 March 1990; revised 8 June 1990) \\ Communicated by A. G. Pakes
}

\begin{abstract}
The paper gives a spectral representation for a class of random fields which are bounded in mean square almost surely. A characterisation of the corresponding spectral measure in the representation is obtained based on Beurling's duality theory and generalised Fourier transforms. A representation for the covariance function of asymptotically stationary random fields is also derived.
\end{abstract}

1991 Mathematics subject classification (Amer. Math. Soc.): 60 G 60, 62 M 15.

\section{Introduction}

Suppose that $\left\{\mathrm{X}(\mathbf{t}), \mathbf{t} \in \mathrm{R}^{n}\right\}$ is a homogeneous complex-valued random field for which $E(\mathrm{X}(\mathrm{t}))=0$ and $E|\mathrm{X}(\mathbf{t})|^{2}<\infty$. Additionally, assume that $\mathrm{X}(\mathrm{t})$ is continuous in mean square and let $R(t)$ denote its covariance function. Then, by Bochner's theorem, $R(t)$ has the representation

$$
\mathbf{R}(\mathbf{t})=\int_{\mathbf{R}^{n}} \mathrm{e}^{-i(\mathbf{t}, \lambda)} d \mathbf{F}(\lambda),
$$

where $F(\lambda)$ is the spectral distribution function of $X(t)$ and $(t, \lambda)$ denotes the inner product of $\mathbf{t}, \lambda \in \mathbf{R}^{n}$. Furthermore, there exists a field $Z(\lambda)$ with orthogonal increments such that for each $\mathbf{t} \in \mathbf{R}^{n}, \mathbf{X}(\mathbf{t})$ has the representation

$$
\mathrm{X}(\mathrm{t})=\int_{\mathbf{R}^{n}} \mathrm{e}^{-i(\mathbf{t}, \lambda)} d \mathrm{Z}(\lambda)
$$

with $E|d \mathrm{Z}(\lambda)|^{2}=d \mathrm{~F}(\lambda)$.

(C) 1992 Australian Mathematical Society 0263-6115/92 \$A2.00+0.00 
This spectral theory for homogeneous random fields was first rigorously established in Yaglom (1957). Although homogeneity (or stationarity) is an important concept, which leads to an elegant theory for analysis of random fields, many significant applications of the theory of random fields, for example in image processing, require the removal of this stationarity condition (see the collection of selected papers in Multidimensional Digital Signal Processing, edited by the Multidimensional Signal Processing Committee, IEEE Press, 1986).

A large class of nonstationary random fields was considered in Wiener (1930), Chapter 3, which can be defined by the condition that

$$
\mathbf{R}(\mathbf{k})=\lim _{\mathbf{T} \rightarrow \infty} \frac{1}{V_{\mathbf{T}}} \int_{C_{\mathbf{T}}(0)} \mathbf{X}(\mathbf{t}+\mathbf{k}) \overline{\mathbf{X}(\mathbf{t})} d \mathbf{t}
$$

exists almost surely for each $\mathbf{k} \in \mathbf{R}^{n}$, where $\mathbf{T}=\left(T_{1}, \ldots, T_{n}\right) \in \mathbf{R}^{n}$,

$$
C_{\mathbf{T}}(\mathbf{y})=\left\{\mathbf{x} \in \mathbf{R}^{n} ;\left|x_{i}-y_{i}\right| \leq T_{i}, i=1, \ldots, n\right\}, \quad \mathbf{y} \in \mathbf{R}^{n},
$$

and $V_{\mathrm{T}}$ is the volume of $C_{\mathrm{T}}(0)$.

Such a class corresponding to $n=1$ (that is, a class of time series) was named the class $S$ in Wiener (1957). Therefore, we shall call $S$ the class of random fields defined by (1.3). A spectral representation for $S$ in the scalar case $(n=1)$ was given in Wiener (1930), Eq. (6.05), p. 155. However, the result, which relies on Cesaro summation procedure, is not as elegant as (1.2). (In fact, it holds only in the sense of integration by parts). A variant of the class $S$, named the class of asymptotically stationary random fields, was investigated in Anh and Lunney (1991). In this latter study, the class of almost harmonizable random fields was shown to be a subclass of the class of asymptotically stationary random fields. Consequently, harmonizable random fields, and therefore, stationary random fields, are all asymptotically stationary. An important example of asymptotically stationary random fields is the class of periodically correlated random fields. Properties of this latter class were shown in Anh and Lunney (1990b) to be useful in characterising the unit pattern in texture analysis. (For a recerit survey on various important classes of nonstationary processes, Rao (1985) and Bhagavan (1985) may be consulted.)

A more general class of random fields which contains $S$ is the class (1.5)

$$
\mathscr{B}=\left\{X(\mathbf{t}) \text { locally in } L^{2} \text { and } \sup _{\mathbf{T}>0} \frac{1}{1+V_{\mathbf{T}}} \int_{C_{\mathrm{T}^{(0)}}}|X(\mathbf{t})|^{2} d \mathbf{t}<\infty \quad \text { a.s. }\right\} \text {. }
$$

Here, $L^{2}$ is the space of quadratically integrable functions on $\mathbf{R}^{n}$ with respect to Lebesgue measure and $X: \mathbf{R}^{n} \rightarrow \mathbf{C}$ is said to be locally in $L^{2}$ if 
$\int_{E}|X(\mathbf{t})|^{2} d \mathbf{t}<\infty$ for every compact set $E$ of $\mathbf{R}^{n}$. It is known that signals with finite power such as those of $\mathscr{B}$ have in general no Fourier transforms. They are harmonically analysed by the so-called generalised Fourier transform used so effectively in Wiener (1930). Several properties for $\mathscr{B}$ with $n=1$ were summarised in Papoulis (1965), Section 13.4. More details for the scalar case can also be found in Wiener (1957). However, a fundamental study of $\mathscr{B}$ for the scalar case $n=1$ was given in Henniger (1970), where Beurling's theory of convolution algebras and ideas from the theory of distributions were employed.

Part of this paper extends the work of Henniger (1970) to random fields. It should be noted that the technique used in proving Theorem 1 of Section 3 is different from that of Henniger (1970). (In fact, the proof of the converse in Henniger's Theorem 3.1 cannot be used for random fields). A spectral representation for the class $\mathscr{B}$ is given in the next section. Here, the representation will be of the form (1.2) with $Z(\lambda)$ as the limit of a generalised Fourier transform. The result is essentially based on the duality theory of Beurling (1964). A characterisation of the field $Z(\lambda)$ in the spectral representation is obtained in Section 3. Based on this characterisation, the distribution function corresponding to the spectral representation obtained and the covariance function of $\mathscr{B}$ are defined. As is well known, the spectral representation and, in particular, the spectral measure and density function, play a dominant role in the filtering and prediction of stationary random processes and fields (see Gihman and Skorohod (1974), Chapter 4). Based on the results developed in this paper, a theory for filtering and prediction of the large class $\mathscr{B}$ is under consideration. In Anh and Lunney (1991), a continuity theorem for the class $S$ is established. This continuity theorem yields the spectral representation for the covariance function $R(\mathbf{k})$ of $S$ as defined by (1.3). However, the result of Anh and Lunney (1991) assumes the continuity of $R(\mathbf{k})$ at the origin. It is known that, even through $X(\mathbf{t})$ can be assumed to be mean square continuous, the covariance function $R(\mathbf{k})$ defined as the limit of (1.3) may not be continuous at any point. With the hindsight of the work developed in Sections 2 and 3, we now derive in Section 4 an alternative representation for $R(\mathbf{k})$ of $S$ without the assumption that $R(0)$ be continuous. This will involve the generalised Fourier transform $Z(\lambda)$ considered in Section 2.

\section{Spectral representation}

We first introduce some notations and give a summary of Beurling's theory as needed in the analysis. Apart from $C_{\mathrm{T}}(\mathbf{y})$ as defined by (1.4), we also put, 
for convenience, $C_{\varepsilon}=\left\{\mathbf{x} \in \mathbf{R}^{n} ; 0<\mathbf{x}<\varepsilon\right\}, \varepsilon \in \mathbf{R}^{n}$.

The volume of $C_{\mathbf{T}}(\mathbf{y})$ is $V_{\mathbf{T}}=2^{n} T_{1} \cdots T_{n}$, while the volut of $C_{\varepsilon}$ is $V_{\varepsilon}=\varepsilon_{1} \cdots \varepsilon_{n}$, where $\varepsilon=\left(\varepsilon_{1}, \ldots, \varepsilon_{n}\right)$. The norm for the class $\mathscr{B}$ is defined as

$$
\|X\|_{\mathscr{B}}=\sup _{\mathbf{T}>0}\left(\frac{1}{1+V_{\mathbf{T}}} \int_{C_{\mathbf{T}}(0)}|X(\mathbf{t})|^{2} d \mathbf{t}\right)^{1 / 2} .
$$

We next consider

$$
\begin{aligned}
& W=\left\{\alpha: \mathbf{R}^{n} \rightarrow \mathbf{R} ; \alpha(t)>0, \quad \text { non-increasing in }|\mathbf{t}|,\right. \\
& \left.\qquad \int_{\mathbf{R}^{n}} \alpha(\mathbf{t}) d \mathbf{t}<\infty \text { and } \alpha(0)=\lim _{\mathbf{t} \rightarrow 0} \alpha(\mathbf{t})<\infty\right\} .
\end{aligned}
$$

Define the norm in $W$ as

$$
N(\alpha)=\alpha(0)+\int_{\mathbf{R}^{n}} \alpha(\mathbf{t}) d \mathbf{t},
$$

and consider $W_{0}=\{\alpha \in W ; N(\alpha)=1\}$. We next define

$$
\begin{aligned}
L^{2}(\alpha(\mathbf{t}) d \mathbf{t}) & =\left\{X: \mathbf{R}^{n} \rightarrow \mathbf{C} ; \int_{\mathbf{R}^{n}}|X(\mathbf{t})|^{2} \alpha(\mathbf{t}) d \mathbf{t}<\infty\right\}, \\
B & =\bigcap_{\alpha \in W_{0}} L^{2}(\alpha(\mathbf{t}) d \mathbf{t}), \\
\|X\|_{B} & =\sup _{\alpha \in W_{0}}\left(\int_{\mathbf{R}^{n}}|X(\mathbf{t})|^{2} \alpha(\mathbf{t}) d \mathbf{t}\right)^{1 / 2} .
\end{aligned}
$$

Then $B$ is a Banach space in the norm (2.2) (see Beurling (1964), Theorem 1). We further define

$$
\begin{aligned}
A & =\bigcup_{\alpha \in W_{0}} L^{2}(d \mathbf{t} / \alpha(\mathbf{t})), \\
\|X\|_{A} & =\inf _{\alpha \in W_{0}}\left(\int_{\mathbf{R}^{n}}|X(\mathbf{t})|^{2} d \mathbf{t} / \alpha(\mathbf{t})\right)^{1 / 2} .
\end{aligned}
$$

Then $A$ is a Banach algebra under addition and convolution with the norm (2.3) and $\left\|X_{1} * X_{2}\right\|_{A} \leq\left\|X_{1}\right\|_{A}\left\|X_{2}\right\|_{A}$ (see Beuling (1964), Theorem 1).

An important result of Beurling (1964) that we require is the following.

THEOREM (Beurling).

(i) $\mathscr{B}=B=A^{*}$, where $A^{*}$ is the dual of $A$ in the Banach space sense;

(ii) each linear functional $X$ on $A$ has the form

$$
X(\varphi)=\int_{\mathbf{R}^{n}} \varphi(\mathbf{t}) \overline{Y(\mathbf{t})} d \mathbf{t}
$$


for a unique $Y$ in $B$ and

$$
\|X\|_{A^{*}}=\sup _{\|\varphi\|_{A}=1}\left|\int_{\mathbf{R}^{n}} \varphi(\mathbf{t}) \overline{Y(\mathbf{t})} d \mathbf{t}\right|=\|Y\|_{B}
$$

(see Beurling (1964), Theorem 2).

The above theory was developed for functions on $\mathbf{R}^{n}$. By considering the random field $\{X(t)\}$ as the class of sample functions $X_{\omega}$ (or simply $X$ ) on a probability space $(\Omega, \mathscr{A}, \mathrm{P})$ (see Gihman and Skorohod (1974), p. 42), then Beurling's theory applies and the results hold almost surely. We shall follows this convention throughout the paper. From Beurling's theorem, it is seen that, for $X \in \mathscr{B}, X \in L^{2}\left(d \mathbf{t} /\left(1+|\mathbf{t}|^{n+1}\right)\right)$ since $1 /\left(1+|\mathbf{t}|^{n+1}\right) \in W$.

Also,

Thus, for large $|\mathbf{t}|$,

$$
\int_{C_{\mathbf{T}}(0)} e^{i(\mathbf{t}, \mathbf{x})} d \mathbf{x}=0\left(|\mathbf{t}|^{-(n+1) / 2}\right) .
$$

$$
\begin{gathered}
\int_{\mathbf{R}^{n}}|X(\mathbf{t})|^{2}\left|\int_{C_{\varepsilon}} e^{i(\mathbf{t}, \mathbf{x})} d \mathbf{x}\right|^{2} d \mathbf{t} \leq c \int_{\mathbf{R}^{n}}|X(\mathbf{t})|^{2} \frac{d \mathbf{t}}{1+|\mathbf{t}|^{n+1}} \\
<\infty ; \text { for some constant } c
\end{gathered}
$$

that is,

$$
X(\mathbf{t}) \int_{C_{\varepsilon}} e^{i(\mathbf{t}, \mathbf{x})} d \mathbf{x} \in L^{2} .
$$

Its Fourier transform is then

$$
Z_{\varepsilon}(\lambda)=\frac{1}{(2 \pi)^{n}} \int_{\mathbf{R}^{n}} X(\mathbf{t})\left(\int_{C_{\varepsilon}} e^{i(\mathbf{t}, \mathbf{x})} d \mathbf{x}\right) e^{i(\mathbf{t}, \lambda)} d \mathbf{t} .
$$

Therefore,

$$
\frac{Z_{\varepsilon}(\lambda)}{V_{\varepsilon}}=\frac{1}{(2 \pi)^{n}} \int_{\mathbf{R}^{n}} X(\mathbf{t})\left(\frac{1}{V_{\varepsilon}} \int_{C_{\varepsilon}} e^{i(\mathbf{t}, \mathbf{x})} d \mathbf{x}\right) e^{i(\mathbf{t}, \lambda)} d \mathbf{t} .
$$

REMARK. The above quantity $Z_{\varepsilon}(\lambda)$ is the generalised Fourier transform of $X(t)$. In the scalar case, this is defined by

$$
G\left(\lambda_{1}\right)-G\left(\lambda_{2}\right)=\frac{1}{2 \pi} \int_{-\infty}^{\infty} \frac{e^{-i \lambda_{1} t}-e^{-i \lambda_{2} t}}{-i t} X(t) d t
$$

(see Papoulis (1965), p. 468).

Therefore,

$$
\begin{aligned}
\frac{G(\lambda+\varepsilon)-G(\lambda-\varepsilon)}{2 \varepsilon} & =\frac{1}{2 \pi} \int_{-\infty}^{\infty} X(t) \frac{\sin \varepsilon t}{\varepsilon t} e^{i \lambda t} d t \\
& =\frac{1}{2 \pi} \int_{-\infty}^{\infty} X(t)\left(\frac{1}{2 \varepsilon} \int_{-\varepsilon}^{\varepsilon} e^{i t x} d x\right) e^{i t \lambda} d t
\end{aligned}
$$

which is of the form (2.4) above. 
For the derivation of the spectral representation, we require the following result due to Henniger (1970).

Lemma 1. Let $\left\{X_{n}\right\}$ be a sequence in $\mathscr{B}$. Consider the following statements:

(a) $\left\{X_{n}\right\}$ is a Cauchy sequence in the weak-star topology of $\mathscr{B}$;

(b) $\left\{X_{n}\right\}$ is a Cauchy sequence in $L^{2}(\alpha(\mathbf{t}) d \mathbf{t})$ for each $\alpha \in W$;

(c) $\left\{X_{n}\right\}$ is a Cauchy sequence in $L^{2}\left(C_{\mathbf{T}}(0)\right)$ for each $\mathbf{T}>0$.

Then $(\mathbf{b}) \Rightarrow$ (c) and $(\mathbf{b}) \Rightarrow(\mathbf{a})$.

If $\left\{X_{n}\right\}$ is a bounded set in $\mathscr{B}$, then $(\mathrm{b}) \Leftrightarrow(\mathrm{c})$. If $X_{n}$ is bounded and any of $(\mathrm{a})-(\mathrm{c})$ holds, then there exists $X$ in $\mathscr{B}$ such that $X_{n} \rightarrow X$ in the respective topology.

Proof. Extension of Theorem 2.1 of Henniger (1970).

Put $X_{\varepsilon}(\mathbf{t})=X(\mathbf{t}) \frac{1}{V_{\varepsilon}} \int_{C_{\varepsilon}} e^{i(\mathbf{t}, \mathbf{x})} d \mathbf{x}$.

Then, as shown above, $X_{\varepsilon} \in L^{2}$ and

$$
\frac{Z_{\varepsilon}(\lambda)}{V_{\varepsilon}}=\frac{1}{(2 \pi)^{n}} \int_{\mathbf{R}^{n}} X_{\varepsilon}(\mathbf{t}) e^{i(\mathbf{t}, \lambda)} d \mathbf{t}
$$

Let $\varphi \in A$. By definition, $\varphi \in L^{2}$. Thus, Parseval's identity implies that, for $\varepsilon \neq 0$,

$$
\int_{\mathbf{R}^{n}} \varphi(\mathbf{t}) X_{\varepsilon}(\mathbf{t}) d \mathbf{t}=\int_{\mathbf{R}^{n}} \hat{\varphi}(\lambda) \frac{Z_{\varepsilon}(\lambda)}{V_{\varepsilon}} d \lambda
$$

where $\hat{\varphi}$ is the Fourier transform of $\varphi$. It is clear that $\left\|X_{\varepsilon}\right\|_{\mathscr{B}} \leq\|X\|_{\mathscr{B}}$ so that $\left\{X_{\varepsilon}\right\}$ is a bounded set in $\mathscr{B}$. Also $X_{\varepsilon} \rightarrow X$ in $L^{2}\left(C_{\mathbf{T}}(0)\right)$ for each $\mathbf{T}>0$ as $\varepsilon \rightarrow 0$ since

$$
\lim _{\varepsilon \rightarrow 0} \frac{1}{V_{\varepsilon}} \int_{C_{\varepsilon}} e^{i(\mathbf{x}, \mathbf{t})} d \mathbf{x}=1
$$

Thus, in view of Lemma 1 above, we get $X_{\varepsilon} \rightarrow X$ in the weak-star topology of $\mathscr{B}$. Consequently, Beurling's theorem implies that

$$
\int_{\mathbf{R}^{n}} \varphi(\mathbf{t}) X_{\varepsilon}(\mathbf{t}) d \mathbf{t} \rightarrow X(\varphi)
$$

and

$$
\widehat{X}(\hat{\varphi})=\lim _{\varepsilon \rightarrow 0} \int_{\mathbf{R}^{n}} \hat{\varphi}(\lambda) \frac{Z_{\varepsilon}(\lambda)}{V_{\varepsilon}} d \lambda=\int_{\mathbf{R}^{n}} \hat{\varphi}(\lambda) d Z(\lambda) .
$$

Eq. (2.5) defines the Fourier transform $\widehat{X}$ of $X \in \mathscr{B}$. It is represented by the measure $d Z$ generated by the generalised Fourier transform $Z_{\varepsilon}$ of $X_{\varepsilon}$. 
For a representation of $X \in \mathscr{B}$, we note that by Plancherel's theorem,

$$
X_{\varepsilon}(\mathbf{t})=\int_{\mathbf{R}^{n}} \frac{Z_{\varepsilon}(\lambda)}{V_{\varepsilon}} e^{-i(\mathbf{t}, \lambda)} d \lambda .
$$

As seen above, $X_{\varepsilon} \rightarrow X$ in any of the three types of convergence specified in Lemma 1. Thus,

$$
X(t)=\lim _{\varepsilon \rightarrow 0} \int_{\mathbf{R}^{n}} \frac{Z_{\varepsilon}(\lambda)}{V_{\varepsilon}} e^{-i(\mathbf{t}, \lambda)} d \lambda
$$

that is,

$$
X(t)=\int_{\mathbf{R}^{n}} e^{-i(\mathbf{t}, \lambda)} d Z(\lambda)
$$

as required.

\section{Characterisation of the spectral measure}

As seen above, $X(\mathbf{t}) \in \mathscr{B}$ has the representation (2.6) where the spectral measure $d Z(\lambda)$ is generated by the generalised Fourier transform $Z_{\varepsilon}$ of $X_{\varepsilon}$ in $L^{2}$. It should be noted from the analysis that the representation (2.6) holds for $X(\mathbf{t})$ in a larger class, namely, $L^{2}\left(d \mathbf{t} /\left(1+|\mathbf{t}|^{n+1}\right)\right)$. In this section, we give a characterisation for $d Z(\lambda)$ corresponding to $X(\mathbf{t}) \in \mathscr{B}$.

We require the following result due to Henniger (1970) for the class

$$
\mathscr{B}_{1}=\left\{X: \mathbf{R} \rightarrow C ; X \text { locally in } L^{2} \text { and } \sup _{T>0} \frac{1}{1+2 T} \int_{-T}^{T}|X(t)|^{2} d t<\infty\right\} \text {. }
$$

LeMMA 2. $X$ belongs to $\mathscr{B}_{1}$ if and only if

$$
S(X)=\sup _{0<\mu<\frac{1}{2}} \int_{\mathbf{R}}|X(t)|^{2} \frac{\sin ^{2} \mu t}{\mu t^{2}} d t<\infty .
$$

Furthermore, there exist constants $k_{1}, k_{2}$ independent of $X$ such that

$$
k_{1}\|X\|_{\mathscr{R}_{1}} \leq S^{1 / 2}(X) \leq k_{2}\|X\|_{\mathscr{B}_{1}} .
$$

Proof. See Henniger (1970), Lemma 3.1.

We now prove

LeMMA 3. $X$ belongs to $\mathscr{B}$ if and only if

$$
S(X)=\sup _{\substack{0<\mu_{j}<1 / 2 \\ j=1, \ldots, n}} \int_{\mathbf{R}^{n}}|X(\mathbf{t})|^{2}\left(\prod_{j=1}^{n} \frac{\sin ^{2} \mu_{j} t_{j}}{\mu_{j} t_{j}^{2}}\right) d \mathbf{t}<\infty .
$$


Proof. Suppose that $X \in \mathscr{B}$. Then, as

$$
\prod_{j=1}^{n}\left(1+2 T_{j}\right) \geq 1+V_{\mathrm{T}},
$$

we get

$$
\begin{aligned}
& \frac{1}{1+2 T_{n}} \int_{-T_{n}}^{T_{n}} \ldots \frac{1}{1+2 T_{1}} \int_{-T_{1}}^{T_{1}}\left|X\left(t_{1}, \ldots, t_{n}\right)\right|^{2} d t_{1} \cdots d t_{n} \\
& \quad \leq \sup _{\mathbf{T}>0} \frac{1}{1+V_{\mathbf{T}}} \int_{C_{\mathbf{T}}(0)}|X(\mathbf{t})|^{2} d \mathbf{t}<\infty .
\end{aligned}
$$

This implies that, for $j=1, \ldots, n$,

$$
\sup _{T_{j}>0} \frac{1}{1+2 T_{j}} \int_{-T_{j}}^{T_{j}}\left|X\left(t_{1}, \ldots, t_{n}\right)\right|^{2} d t_{j}<\infty .
$$

Therefore, in view of Lemma 2 above and for $j=1, \ldots, n$,

$$
\begin{aligned}
& \sup _{0<\mu_{j}<1 / 2} \int_{-\infty}^{\infty}\left|X\left(t_{1}, \ldots, t_{n}\right)\right|^{2} \frac{\sin ^{2} \mu_{j} t_{j}}{\mu_{j} t_{j}^{2}} d t_{j} \\
& \quad \leq k_{j} \sup _{T_{j}>0} \frac{1}{1+2 T_{j}} \int_{-T_{j}}^{T_{j}}\left|X\left(t_{1}, \ldots, t_{n}\right)\right|^{2} d t_{j}
\end{aligned}
$$

for some constant $k_{j}$ independent of $X$. Now, for $0<\mu_{2}<1 / 2$,

$$
\begin{aligned}
\int_{-\infty}^{\infty} & \left(\sup _{0<\mu_{1}<1 / 2} \int_{-\infty}^{\infty}\left|X\left(t_{1}, \ldots, t_{n}\right)\right|^{2} \frac{\sin ^{2} \mu_{1} t_{1}}{\mu_{1} t_{1}^{2}} d t_{1}\right) \frac{\sin ^{2} \mu_{2} t_{2}}{\mu_{2} t_{2}^{2}} d t_{2} \\
& \leq k_{1} \int_{-\infty}^{\infty}\left(\sup _{T_{1}>0} \frac{1}{1+2 T_{1}} \int_{-T_{1}}^{T_{1}}\left|X\left(t_{1}, \ldots, t_{n}\right)\right|^{2} d t_{1}\right) \frac{\sin ^{2} \mu_{2} t_{2}}{\mu_{2} t_{2}^{2}} d t_{2} \\
& \leq k_{1} \sup _{T_{1}>0} \frac{1}{1+2 T_{1}} \int_{-T_{1}}^{T_{1}}\left(\sup _{0<\mu_{2}<1 / 2} \int_{-\infty}^{\infty}\left|X\left(t_{1}, \ldots, t_{n}\right)\right|^{2} \frac{\sin ^{2} \mu_{2} t_{2}}{\mu_{2} t_{2}^{2}} d t_{2}\right) d t_{1} \\
& \leq k_{1} k_{2} \sup _{T_{1}>0} \frac{1}{1+2 T_{1}} \int_{-T_{1}}^{T_{1}} \sup _{T_{2}>0} \int_{-T_{2}}^{T_{2}}\left|X\left(t_{1}, \ldots, t_{n}\right)\right|^{2} d t_{2} d t_{1}
\end{aligned}
$$

for some constant $k_{2}$ in view of (3.5). 
Repeating the argument will lead to

$$
\begin{aligned}
& \sup _{\substack{0<\mu_{j}<1 / 2 \\
j=1, \ldots, n}} \int_{\mathbf{R}^{n}}\left|X\left(t_{1}, \ldots, t_{n}\right)\right|^{2}\left(\prod_{j=1}^{n} \frac{\sin ^{2} \mu_{j} t_{j}}{\mu_{j} t_{j}^{2}}\right) d t_{1} \cdots d t_{n} \\
& \leq k_{1} \cdots k_{n} \sup _{T_{1}>0} \frac{1}{1+2 T_{1}} \\
& \quad \times \int_{-T_{1}}^{T_{1}} \cdots \sup _{T_{n}>0} \frac{1}{1+2 T_{n}} \int_{-T_{n}}^{T_{n}}\left|X\left(t_{1}, \ldots, t_{n}\right)\right|^{2} d t_{1} \cdots d t_{n} \\
& <\infty \text { for some constants } k_{1}, \ldots, k_{n}
\end{aligned}
$$

Conversely, suppose that for some $\mathbf{T}^{\prime}=\left(T_{1}^{\prime}, \ldots, T_{n}^{\prime}\right)$,

$$
\frac{1}{1+V_{\mathbf{T}^{\prime}}} \int_{C_{\mathbf{T}^{\prime}}(0)}|X(\mathbf{t})|^{2} d \mathbf{t}>M, \quad M>0 .
$$

We can assume that $T_{j}^{\prime} \geq 2, j=1, \ldots, n$. Take $\mu_{j}=\pi /\left(4 T_{j}^{\prime}\right), j=$ $1, \ldots, n$. Then, for $j=1, \ldots, n, 0<\mu_{j}<1 / 2$ and $\sin ^{2} \mu_{j} t_{j} \geq 1 / 2$ for $T_{j}^{\prime} \leq t \leq 2 T_{j}^{\prime}$.

Also from (3.6),

$$
\frac{1}{1+V_{\mathbf{T}}} \int_{C_{\mathbf{T}}(0)}|X(\mathbf{t})|^{2} d \mathbf{t}>\frac{M}{2^{n}} \quad \text { for } \quad \mathbf{T}_{1}^{\prime} \leq \mathbf{T} \leq 2 \mathbf{T}_{1}^{\prime} .
$$

Thus, for $\mathbf{T}_{1}^{\prime} \leq \mathbf{T} \leq 2 \mathbf{T}_{1}^{\prime}$,

$$
\begin{aligned}
\int_{T_{1}}^{2 T_{1}} \cdots \int_{T_{n}}^{2 T_{n}}|X(\mathbf{t})|^{2}\left(\prod_{j=1}^{n} \frac{\sin ^{2} \mu_{j} t_{j}}{\mu_{j} t_{j}^{2}}\right) d t & \geq \frac{1}{(2 \pi)^{n}}\left(\prod_{j=1}^{n} \frac{1}{T_{j}^{\prime}}\right) \int_{C_{\mathbf{T}}(0)}|X(\mathbf{t})|^{2} d \mathbf{t} \\
& >\frac{1}{(2 \pi)^{n}}\left(\prod_{j=1}^{n} \frac{1}{T_{j}^{\prime}}\right)\left(1+V_{\mathrm{T}}\right) \frac{M}{2^{n}} \\
& >\frac{1}{(2 \pi)^{n}}\left(\prod_{j=1}^{n} \frac{1}{T_{j}^{\prime}}\right) V_{\mathbf{T}} \frac{M}{2^{n}} \\
& \geq \frac{1}{(2 \pi)^{n}} \frac{2^{n} T_{1}^{\prime} \cdots T_{n}^{\prime} M}{T_{1}^{\prime} \cdots T_{n}^{\prime}} \frac{M}{2^{n}} \\
& =\frac{M}{(2 \pi)^{n}} .
\end{aligned}
$$

Consequently, if the mean square of $X$ is unbounded, then $S(X)=\infty$, a contradiction.

We now characterise the spectral measure $d Z$ for $X \in \mathscr{B}$. 
THEOREM 1. The field $Z(\lambda)$ of (2.5) and (2.6) generates a spectral measure $d Z$ in $\widehat{\mathscr{B}}$, the space of Fourier transforms of $X \in \mathscr{B}$, if and only if it is locally in $L^{2}$ and satisfies

$$
D(Z)=\sup _{0<\varepsilon<1} \frac{1}{V_{\varepsilon}} \int_{\mathbf{R}^{n}}\left|Z_{\varepsilon}(\lambda)\right|^{2} d \lambda<\infty .
$$

Proof. Since $Z_{\varepsilon}(\lambda) / V_{\varepsilon}$ is the Fourier transform of $X_{\varepsilon}(t)$, we get

$$
\frac{1}{(2 \pi)^{n}} \int_{\mathbf{R}^{n}}\left|X_{\varepsilon}(\mathbf{t})\right|^{2} d \mathbf{t}=\int_{\mathbf{R}^{n}}\left|\frac{Z_{\varepsilon}(\lambda)}{V_{\varepsilon}}\right|^{2} d \lambda .
$$

But $\left|e^{i \varepsilon_{j} t_{j}}-1\right|^{2}=4 \sin ^{2} \varepsilon_{j} t_{j} / 2$. Therefore, we can rewrite equation (3.8) as

$$
\frac{1}{\pi^{n}} \int_{\mathbf{R}^{n}}|X(\mathbf{t})|^{2} \prod_{j=1}^{n}\left(\frac{\sin ^{2} \varepsilon_{j} t_{j} / 2}{\varepsilon_{j} t_{j}^{2} / 2}\right) d \mathbf{t}=\frac{1}{V_{\varepsilon}} \int_{\mathbf{R}^{n}}\left|Z_{\varepsilon}(\lambda)\right|^{2} d \lambda .
$$

The left hand side of (3.9) is bounded for $0<\varepsilon<1$ by Lemma 3, taking $\mu_{j}=\varepsilon_{j} / 2, j=1, \ldots, n$. Consequently, (3.7) holds.

Conversely, suppose that $Z_{\varepsilon}$ is a field locally in $L^{2}$ and satisfies (3.7). Then, using Plancherel's theorem, we can define a function $X(\mathbf{t}, \varepsilon)$ such that $X(\mathbf{t}, \varepsilon)$ is locally in $L^{2}$ for each $\varepsilon>0$ and $X(\mathbf{t}, \varepsilon) \int_{C_{\varepsilon}} e^{i(\mathbf{t}, \mathbf{x})} d \mathbf{x}$ is the inverse transform of $Z_{\varepsilon}(\lambda)$, that is,

$$
Z_{\varepsilon}(\lambda)=\frac{1}{(2 \pi)^{n}} \lim _{\mathbf{T} \rightarrow \infty} \int_{C_{\mathbf{T}}(0)}\left(\prod_{j=1}^{n} \frac{e^{i \varepsilon_{j} t_{j}}-1}{i t_{j}}\right) X(\mathbf{t}, \varepsilon) e^{i(t, \lambda)} d \mathbf{t} .
$$

We want to show that $X(\mathbf{t}, \varepsilon)=\int_{\mathbf{R}^{n}} e^{-i(\mathbf{t}, \mathbf{u})} d Z(\mathbf{u})=X(\mathbf{t})$, independent of $\varepsilon$. In fact, put

$$
g(\mathbf{T})=\int_{C_{\mathbf{T}}(0)} \prod_{j=1}^{n}\left(\frac{e^{i t_{j}\left(\varepsilon_{j}+\lambda_{j}\right)}-e^{i t_{j} \lambda_{j}}}{i t_{j}}\right)\left(\int_{\mathbf{R}^{n}} e^{-i(\mathbf{t}, \mathbf{u})} d Z(\mathbf{u})\right) d \mathbf{t} .
$$

Then

$$
\begin{aligned}
g(\mathbf{T}) & =\int_{\mathbf{R}^{n}} \int_{C_{\mathbf{T}}(0)} \prod_{j=1}^{n}\left(\frac{e^{i t_{j}\left(\varepsilon_{j}+\lambda_{j}-u_{j}\right)}-e^{i t_{j}\left(\lambda_{j}-u_{j}\right)}}{i t_{j}}\right) d \mathbf{t} d Z(\mathbf{u}) \\
& =2^{n} \int_{\mathbf{R}^{n}} h(\mathbf{u}, \mathbf{T}) d Z(\mathbf{u}),
\end{aligned}
$$

where

$$
h(\mathbf{u}, \mathbf{T})=\prod_{j=1}^{n} \int_{0}^{T_{j}} \frac{\sin t_{j}\left(\varepsilon_{j}+\lambda_{j}-u_{j}\right)-\sin t_{j}\left(\lambda_{j}-u_{j}\right)}{t_{j}} d t_{j} .
$$


If we denote by $C$ the cube $\left\{\mathbf{u} \in \mathbf{R}^{n} ; \lambda<\mathbf{u}<\lambda+\varepsilon\right\}$, then

$$
\begin{aligned}
g(\mathbf{T}) & =2^{n}\left(\int_{C^{c}}+\int_{\partial C}+\int_{C}\right) h(\mathbf{u}, \mathbf{T}) d Z(\mathbf{u}) \\
& =2^{n}\left(I_{1}+I_{2}+I_{3}\right),
\end{aligned}
$$

where $C^{c}$ is the complement of $C$ and $\partial C$ its boundary.

Since $C$ is a continuity set of $Z(\mathbf{u}), I_{2}=0$. Also,

$$
\lim _{\mathbf{T} \rightarrow \infty} h(\mathbf{u}, \mathbf{T})= \begin{cases}0, & \mathbf{u} \notin \bar{C} \\ \pi^{n}, & \mathbf{u} \in C\end{cases}
$$

using the result that

$$
\int_{0}^{\infty} \frac{\sin a t}{t} d t= \begin{cases}\pi / 2, & a>0 \\ 0, & a=0 \\ -\pi / 2, & a<0\end{cases}
$$

(see Lukacs (1970) p. 31). Consequently, in view of the dominated convergence theorem, we get

$$
\begin{aligned}
& \lim _{\mathbf{T} \rightarrow \infty} I_{1}=\int_{C^{c}}\left(\lim _{\mathbf{T} \rightarrow \infty} h(\mathbf{u}, \mathbf{T})\right) d Z(\mathbf{u})=0, \\
& \lim _{\mathbf{T} \rightarrow \infty} I_{3}=\int_{C}\left(\lim _{\mathbf{T} \rightarrow \infty} h(\mathbf{u}, \mathbf{T})\right) d Z(\mathbf{u})=\pi^{n}(Z(\lambda+\varepsilon)-Z(\lambda))
\end{aligned}
$$

so that

$$
\lim _{\mathbf{T} \rightarrow \infty} g(\mathbf{T})=Z_{\varepsilon}(\lambda)
$$

It then follows that $X(\mathbf{t}, \varepsilon)=\int_{\mathbf{R}^{n}} e^{-i(\mathbf{t}, \mathbf{u})} d Z(\mathbf{u})$ a.e.

The Parseval identity now implies

$$
\int_{\mathbf{R}^{n}}\left|Z_{\varepsilon}(\lambda)\right|^{2} d \lambda=\frac{1}{(2 \pi)^{n}} \int_{\mathbf{R}^{n}}|X(\mathbf{t})|^{2} \prod_{j=1}^{n} \frac{4 \sin ^{2} \varepsilon_{j} t_{j} / 2}{t_{j}^{2}} d \mathbf{t},
$$

that is,

$$
\frac{1}{V_{\varepsilon}} \int_{\mathbf{R}^{n}}\left|Z_{\varepsilon}(\lambda)\right|^{2} d \lambda=\frac{1}{\pi^{n}} \int_{\mathbf{R}^{n}}|X(\mathbf{t})|^{2} \prod_{j=1}^{n} \frac{\sin ^{2} \varepsilon_{j} t_{j} / 2}{\varepsilon_{j} t_{j}^{2} / 2} d \mathbf{t} .
$$

Condition (3.7) then yields that $X \in \mathscr{B}$ in view of Lemma 3.

\section{Representation for the covariance function}

In view of Theorem 1, the distribution function corresponding to the representation (2.6) may be defined as

$$
F(\mathbf{u})=\sup _{0<\varepsilon<1} \frac{1}{V_{\varepsilon}} \int_{-\infty}^{u_{n}} \cdots \int_{-\infty}^{u_{1}}\left|Z_{\varepsilon}(\lambda)\right|^{2} d \lambda_{1} \cdots d \lambda_{n}
$$


for $\mathbf{u}=\left(u_{1}, \ldots, u_{n}\right) \in \mathbf{R}^{n}$. This definition suggests a way to define the covariance function of $\mathscr{B}$ as

$$
R(\mathbf{k})=\int_{\mathbf{R}^{n}} e^{-i(\mathbf{k}, \mathbf{u})} d F(\mathbf{u})
$$

if the transform exists.

For Wiener's class $S$, which is a subclass of $\mathscr{B}$, result (4.2) has a more definite setting. It is proved in Anh and Lunney (1991) that the representation (4.2) holds under some conditions including the continuity of $R(0)$. In this section, we shall remove the latter restriction. An alternative representation for $R(\mathbf{k})$ is derived based on the generalised Fourier transform $Z_{\varepsilon}(\lambda)$ of the previous sections.

We shall require the following

Wiener's TAuberian Theorem. Suppose that $\psi \in L^{\infty}\left(\mathbf{R}^{n}\right), f \in L^{1}\left(\mathbf{R}^{n}\right)$, $\hat{f}(\lambda) \neq 0$ for every $\lambda \in \mathbf{R}^{n}$, and

$$
\lim _{|\mathbf{t}| \rightarrow \infty}(f * \psi)(\mathbf{t})=a \hat{f}(0)
$$

Then $\lim _{|\mathbf{t}| \rightarrow \infty}(g * \psi)(\mathbf{t})=a \hat{g}(0)$ for every $g \in L^{1}\left(\mathbf{R}^{n}\right)$.

Proof. See Rudin (1973), pp. 211-212.

Let $\psi$ be as given in the above theorem. Put

$$
\begin{gathered}
t_{j}=e^{\xi_{j}}, \quad T_{j}=e^{\eta_{j}}, \quad \varepsilon_{j}=e^{-\eta_{j}}, \quad j=1, \ldots, n \\
\varphi(\mathbf{t})=\psi(\xi), \quad \mathbf{t}, \xi \in \mathbf{R}^{n} .
\end{gathered}
$$

Then

$$
\begin{aligned}
& \frac{1}{T_{1} \cdots T_{n}} \int_{0}^{T_{n}} \cdots \int_{0}^{T_{1}} \varphi(\mathbf{t}) d t_{1} \cdots d t_{n} \\
& =\frac{1}{e^{\eta_{1}+\cdots+\eta_{n}}} \int_{-\infty}^{\eta_{n}} \cdots \int_{-\infty}^{\eta_{1}} e^{\xi_{1}+\cdots+\xi_{n}} \psi(\xi) d \xi_{1} \cdots d \xi_{n}, \\
& \left(\frac{2}{n}\right)^{n} \int_{0}^{\infty} \cdots \int_{0}^{\infty} \varphi(\mathbf{t})\left(\prod_{j=1}^{n} \frac{\sin ^{2} \varepsilon_{j} t_{j}}{\varepsilon_{j} t_{j}^{2}}\right) d \mathbf{t} \\
& \quad=\int_{-\infty}^{\infty} \cdots \int_{-\infty}^{\infty}\left(\prod_{j=1}^{n} \frac{2}{\pi} \frac{\sin ^{2}\left(e^{\xi_{j}-\eta_{j}}\right)}{e^{\xi_{j}-\eta_{j}}}\right) \psi(\xi) d \xi_{1} \cdots d \xi_{n} .
\end{aligned}
$$


Define

$$
\begin{aligned}
& f(\xi)=\prod_{j=1}^{n} \frac{2}{\pi} e^{\xi_{j}} \sin ^{2}\left(e^{-\xi_{j}}\right), \quad \xi \in \mathbf{R}^{n}, \\
& g(\xi)= \begin{cases}e^{-\left(\xi_{1}+\cdots+\xi_{n}\right)}, & 0<\xi_{j}<\infty, j=1, \ldots, n \\
0, & \text { otherwise. }\end{cases}
\end{aligned}
$$

Then,

$$
\begin{aligned}
\left.\int_{\mathbf{R}^{n}} f(\xi) d \xi\right) & =\prod_{j=1}^{n} \int_{-\infty}^{\infty} \frac{2}{\pi} e^{\xi_{j}} \sin ^{2}\left(e^{-\xi_{j}}\right) d \xi_{j} \\
& =\prod_{j=1}^{n} \int_{0}^{\infty} \frac{\sin ^{2} x_{j}}{x_{j}^{2}} d x_{j}=1 \\
\int_{\mathbf{R}^{n}} g(\xi) d \xi & =\prod_{j=1}^{n} \int_{0}^{\infty} e^{-\xi_{j}} d \xi_{j}=1 .
\end{aligned}
$$

Also,

$$
\lim _{\varepsilon \rightarrow 0}\left(\frac{2}{\pi}\right)^{n} \int_{0}^{\infty} \cdots \int_{0}^{\infty} \varphi(\mathbf{t})\left(\prod_{j=1}^{n} \frac{\sin ^{2} \varepsilon_{j} t_{j}}{\varepsilon_{j} t_{j}^{2}}\right) d \mathbf{t}=\lim _{\eta \rightarrow \infty} \int_{\mathbf{R}^{n}} f(\eta-\xi) \psi(\xi) d \xi
$$

(4.2) $\lim _{\mathbf{T} \rightarrow \infty} \frac{1}{T_{1} \cdots T_{n}} \int_{0}^{T_{n}} \cdots \int_{0}^{T_{1}} \varphi(\mathbf{t}) d t_{1} \cdots d t_{n}=\lim _{\eta \rightarrow \infty} \int_{\mathbf{R}^{n}} g(\eta-\xi) \psi(\xi) d \xi$.

As shown by Wiener (1958), pp. 142-143, for each $j=1, \ldots, n$, $(2 / \pi) \exp \left(\xi_{j}\right) \sin ^{2} \exp \left(-\xi_{j}\right) \in L^{1}(\mathbf{R})$ and its Fourier transform does not vanish on $\mathbf{R}$. Thus, $f \in L^{1}\left(\mathbf{R}^{n}\right)$ and $\hat{f}(\lambda) \neq 0, \lambda \in \mathbf{R}^{n}$. Consequently, the two limits in (4.1) and (4.2) assume the same value, if they exist, by Wiener's Tauberian theorem as stated above.

By choosing $\varphi(\mathbf{t})=|X(\mathbf{t})|^{2}, X \in S$, the above analysis yields immediately

$$
\lim _{\mathbf{T} \rightarrow \infty} \frac{1}{V_{\mathbf{T}}} \int_{C_{\mathbf{T}}(0)}|X(\mathbf{t})|^{2} d \mathbf{t}=\lim _{\varepsilon \rightarrow 0} \frac{1}{\pi^{n}} \int_{\mathbf{R}^{n}}|X(\mathbf{t})|^{2} \prod_{j=1}^{n} \frac{\sin ^{2} \varepsilon_{j} t_{j}}{\varepsilon_{j} t_{j}^{2}} d \mathbf{t} .
$$

In conjunction with (3.9), we then have

$$
\lim _{\mathbf{T} \rightarrow \infty} \frac{1}{V_{\mathbf{T}}} \int_{C_{\mathbf{T}}(0)}|X(\mathbf{t})|^{2} d \mathbf{t}=\lim _{\varepsilon \rightarrow 0} \frac{1}{V_{\varepsilon}} \int_{\mathbf{R}^{n}}\left|Z_{\varepsilon}(\lambda)\right|^{2} d \lambda .
$$

REMARK. The quantity on the right hand side of (4.3) is known as the quadratic variation of the integrated Fourier transform $Z_{\varepsilon}$. Result (4.3) states that the quadratic variation of the integrated Fourier transform of a 
field is equal to its mean square value. This important result is known as Wiener's theorem of quadratic variation in the scalar case. (See Lee (1960), pp. 85-92, for a study of the quadratic variation of several important classes of time series).

It should be noted that the left hand side of $(4.3)$ is $R(0)\left(=\int_{\mathbf{R}^{n}} d F(\lambda)\right)$ and result (4.3) in fact motivaties the definition of the distribution function $F(\mathbf{u})$ given in (4.1) above. The usual relationship between the covariance function and the distribution function then suggests that

$$
{ }^{-} R(\mathbf{k})=\lim _{\varepsilon \rightarrow 0} \frac{1}{V_{\varepsilon}} \int_{\mathbf{R}^{n}} e^{-i(\mathbf{k}, \lambda)}\left|Z_{\varepsilon}(\lambda)\right|^{2} d \lambda .
$$

We now prove that this is indeed the case for Wiener's class $S$ and the result does not require the continuity of $R(\mathbf{k})$.

Theorem 2. Let $X \in S$ and $R(\mathbf{k})$ be defined by (1.3). Then $R(\mathbf{k})$ has the representation (4.4).

Proof. We have

$$
\begin{aligned}
R(\mathbf{k})=\lim _{\mathbf{T} \rightarrow \infty} \frac{1}{V_{\mathbf{T}}} \int_{C_{\mathbf{T}}(0)} X(\mathbf{t}+\mathbf{k}) \overline{X(\mathbf{t})} d \mathbf{t} & \\
=\frac{1}{4} \lim _{\mathbf{T} \rightarrow \infty} \frac{1}{V_{\mathbf{T}}} \int_{C_{\mathbf{T}}(0)}(\mid X(\mathbf{t} & +\mathbf{k})+\left.X(\mathbf{t})\right|^{2}-|X(\mathbf{t}+\mathbf{k})-X(\mathbf{t})|^{2} \\
& +i|X(\mathbf{t}+\mathbf{k})+i X(\mathbf{t})|^{2} \\
& \left.-i|X(\mathbf{t}+\mathbf{k})-i X(\mathbf{t})|^{2}\right) d \mathbf{t}
\end{aligned}
$$

Define the generalised Fourier transform $Z_{\varepsilon}$ corresponding to $X(\mathbf{t})$, which exists, as in Section 2, and let the generalised Fourier transform corresponding to $X(\mathbf{t}+\mathbf{k})$ be $Z_{\varepsilon, \mathbf{k}}(\lambda)$. Then, using (4.3),

$$
\begin{aligned}
\lim _{\mathbf{T} \rightarrow \infty} & \frac{1}{V_{\mathbf{T}}} \int_{C_{\mathbf{T}}(0)}|X(\mathbf{t}+\mathbf{k})+\alpha X(\mathbf{t})|^{2} d \mathbf{t} \\
\quad & \lim _{\varepsilon \rightarrow 0} \frac{1}{V_{\varepsilon}} \int_{\mathbf{R}^{n}}\left|Z_{\varepsilon, k}(\lambda)+\alpha Z_{\varepsilon}(\lambda)\right|^{2} d \lambda \text { for }|\alpha|=1 \\
& =\lim _{\varepsilon \rightarrow 0} \frac{1}{V_{\varepsilon}} \int_{\mathbf{R}^{n}}\left|Z_{\varepsilon, k}(\lambda)+\alpha Z_{\varepsilon}(\lambda)+e^{-i(\mathbf{k}, \lambda)} Z_{\varepsilon}(\lambda)-e^{-i(\mathbf{k}, \lambda)} Z_{\varepsilon}(\lambda)\right|^{2} d \lambda \\
& =\lim _{\varepsilon \rightarrow 0} \frac{1}{V_{\varepsilon}} \int_{\mathbf{R}^{n}}\left|Z_{\varepsilon, \mathbf{k}}(\lambda)-e^{-i(\mathbf{k}, \lambda)} Z_{\varepsilon}(\lambda)+\left(\alpha+e^{-i(\mathbf{k}, \lambda)}\right) Z_{\varepsilon}(\lambda)\right|^{2} d \lambda
\end{aligned}
$$


Applying Minkowski's inequality to the right hand side of (4.6), we get

$$
\begin{aligned}
& \left(\lim _{\mathbf{T} \rightarrow \infty} \frac{1}{V_{\mathbf{T}}} \int_{C_{\mathbf{T}}(0)}|X(\mathbf{t}+\mathbf{k})+\alpha X(\mathbf{t})|^{2} d \mathbf{t}\right)^{1 / 2} \\
& \leq\left(\lim _{\varepsilon \rightarrow 0} \frac{1}{V_{\varepsilon}} \int_{\mathbf{R}^{n}}\left|Z_{\varepsilon, \mathbf{k}}(\lambda)-e^{-i(\mathbf{k}, \lambda)} Z_{\varepsilon}(\lambda)\right|^{2} d \lambda\right)^{1 / 2} \\
& \quad+\left(\lim _{\varepsilon \rightarrow 0} \frac{1}{V_{\varepsilon}} \int_{\mathbf{R}^{n}}\left|\alpha+e^{-i(\mathbf{k}, \lambda)}\right|^{2}\left|Z_{\varepsilon}(\lambda)\right|^{2} d \lambda\right)^{1 / 2}
\end{aligned}
$$

Now, using the transformation $\mathbf{t}+\mathbf{k} \rightarrow \mathbf{t}$,

$$
\begin{aligned}
Z_{\varepsilon, \mathbf{k}}(\lambda) & =\frac{1}{(2 \pi)^{n}} \int_{\mathbf{R}^{n}} X(\mathbf{t}+\mathbf{k})\left(\prod_{j=1}^{n} \frac{2 \sin \varepsilon_{j} t_{j}}{t_{j}} e^{i t_{j} \lambda_{j}}\right) d \mathbf{t} \\
& =\frac{1}{(2 \pi)^{n}} \int_{\mathbf{R}^{n}} X(\mathbf{t})\left(\prod_{j=1}^{n} \frac{2 \sin \varepsilon_{j}\left(t_{j}-k_{j}\right)}{t_{j}-k_{j}} e^{i\left(t_{j}-k_{j}\right) \lambda_{j}}\right) d \mathbf{t} .
\end{aligned}
$$

Also,

$$
e^{-i(\mathbf{k}, \lambda)} Z_{\varepsilon}(\lambda)=\frac{1}{(2 \pi)^{n}} \int_{\mathbf{R}^{n}} X(\mathbf{t})\left(\prod_{j=1}^{n} \frac{2 \sin \varepsilon_{j} t_{j}}{t_{j}} e^{i\left(t_{j}-k_{j}\right) \lambda_{j}}\right) d \mathbf{t} .
$$

Therefore,

$$
\begin{aligned}
Z_{\varepsilon, \mathbf{k}}(\lambda)-e^{-i(\mathbf{k}, \lambda)} Z_{\varepsilon}(\lambda) & \\
= & \frac{1}{\pi^{n}} \int_{\mathbf{R}^{n}} X(\mathbf{t}) \prod_{j=1}^{n}\left(\left(\frac{\sin \varepsilon_{j}\left(t_{j}-k_{j}\right)}{t_{j}-k_{j}}-\frac{\sin \varepsilon_{j} t_{j}}{t_{j}}\right) e^{i\left(t_{j}-k_{j}\right) \lambda_{j}}\right) d \mathbf{t} .
\end{aligned}
$$

Parseval's identity then yields

$$
\begin{aligned}
& \frac{1}{2^{n}} \int_{\mathbf{R}^{n}}\left|Z_{\varepsilon, \mathbf{k}}(\lambda)-e^{-i(\mathbf{k}, \lambda)} Z_{\varepsilon}(\lambda)\right|^{2} d \lambda \\
& \quad=\frac{1}{\pi^{n}} \int_{\mathbf{R}^{n}}|X(\mathbf{t})|^{2} \prod_{j=1}^{n}\left(\frac{\sin \varepsilon_{j}\left(t_{j}-k_{j}\right)}{t_{j}-k_{j}}-\frac{\sin \varepsilon_{j} t_{j}}{t_{j}}\right)^{2} d \mathbf{t} .
\end{aligned}
$$

As shown in Wiener (1958), pp. 157-158,

$$
\left|\frac{\sin \varepsilon_{j}\left(t_{j}-k_{j}\right)}{t_{j}-k_{j}}-\frac{\sin \varepsilon_{j} t_{j}}{t_{j}}\right| \leq \frac{8 \varepsilon_{j}\left|k_{j}\right|}{\left|t_{j}\right|+\left|k_{j}\right|} .
$$

Also, from Beurling's theorem, it is seen that, for $X \in S, X \in$ $L^{2}\left(d \mathbf{t} /\left(1+|\mathbf{t}|^{2 n}\right)\right)$ since $1 /\left(1+|\mathbf{t}|^{2 n}\right) \in W$. Consequently, the right hand 
side of $(4.8)$ is $0\left(\varepsilon_{1}^{2} \cdots \varepsilon_{n}^{2}\right)$. This result together with (4.7), yields

$$
\begin{aligned}
& \left(\lim _{\mathbf{T} \rightarrow \infty} \frac{1}{V_{\mathbf{T}}} \int_{C_{\mathbf{T}}(0)}|X(\mathbf{t}+\mathbf{k})+\alpha X(\mathbf{t})|^{2} d \mathbf{t}\right)^{1 / 2} \\
& \quad=\lim _{\varepsilon \rightarrow 0}\left\{0\left(\varepsilon_{1}^{1 / 2} \cdots \varepsilon_{n}^{1 / 2}\right)+\left(\frac{1}{V_{\varepsilon}} \int_{\mathbf{R}^{n}}\left|\alpha+e^{-i(\mathbf{k}, \lambda)}\right|^{2}\left|Z_{\varepsilon}(\lambda)\right|^{2} d \lambda\right)^{1 / 2}\right\} \\
& \quad=\lim _{\varepsilon \rightarrow 0}\left(\frac{1}{V_{\varepsilon}} \int_{\mathbf{R}^{n}}\left(2+\alpha e^{i(\mathbf{k}, \lambda)}+\bar{\alpha} e^{-i(\mathbf{k}, \lambda)}\right)\left|Z_{\varepsilon}(\lambda)\right|^{2} d \lambda\right)^{1 / 2} .
\end{aligned}
$$

If we now take $\alpha=1,-1, i,-i$ successively in (4.9) and combine the four results in accordance with (4.5), the representation (4.4) will be obtained.

\section{Acknowledgement}

The authors are grateful to the referee for his constructive comments on the paper.

\section{References}

V. V. Anh and K. E. Lunney (1991), 'Covariance function and ergodicity of asymptotically stationary random fields', Bull. Austral. Math. Soc. 44, 49-62.

V. V. Anh and K. E. Lunney (1990), 'Covariance function of periodically correlated random fields', QUT Working Paper in Mathematics 7/90.

A. Beurling (1964), 'Construction and analysis of some convolution algebras', Annales de l'Institut Fourier 14, 1-32.

C. S. K. Bhagavan (1985), On nonstationary time series, in: Handbook of Statistics (eds. E. J. Hannan, P. R. Krishnaiah and M. M. Rao), Vol. 5, (Elsevier) pp. 311-320.

I. I. Gihman and A. V. Skorohod (1974), The theory of stochastic processes I (Springer, Berlin).

J. Henniger (1970), 'Functions of bounded mean square and generalized Fourier-Stieltjes transforms', Canad. J. Math. 22, 1016-1034.

Y. W. Lee (1960), Statistical theory of communication (Wiley, New York).

E. Lukacs (1970), Characteristic functions (Griffin, London).

A. Papoulis (1965), Probability random variables, and stochastic processes (McGraw-Hill, New York).

M. M. Rao (1985), 'Harmonizable, Cramer, and Karhunen classes of processes', in: Handbook of Statistics (eds. E. J. Hannan, P. R. Krishnaiah and M. M. Rao), Vol. 5, (Elsevier) pp. 279-310.

W. Rudin (1973), Functional analysis (McGraw-Hill, New York).

N. Wiener (1930), 'Generalized harmonic analysis', Acta Math. 55, 117-258. 
N. Wiener (1958), The Fourier integral and certain of its applications (Dover).

A. M. Yaglom (1957), 'Some classes of random fields in $n$-dimensional space, related to stationary random processes', Theory Probab. Appl. 2, 273-340.

School of Mathematics

Queensland University of Technology

GPO Box 2434

Brisbane

Queensland 4001

Australia 